\title{
Review of Julie Spray's (2020) The Children in Child Health: Negotiating Young Lives and Health in New Zealand. Rutgers University Press.
}

\author{
Adriano De Francesco \\ Doctoral Candidate in Anthropology, The University of Auckland
}

Children as social actors - as co-producers of the cultural scripts and material conditions framing their social destinies and, most crucially for this book's purposes, their health - are the protagonists of Julie Spray's penetrating ethnography which represents the culmination of her doctoral research at the University of Auckland.

Spray's one-year ethnographic fieldwork saw her almost daily presence at Tūrama School (a pseudonym) in Papakura, a southern suburb of Auckland, in classrooms of children between eight and twelve years old. The result is an exquisitely crafted monograph in social-cultural anthropology that sits at the vertex of several lines of research, illuminating issues of topical interest for a variety of disciplines. Dominance and social stratification, agency and resistance, emergent peer cultures, identity in the school setting, child health policy: Spray's research skilfully weaves together all these macro-themes and, in doing so, it not only represents a relevant addition to the legacy of anthropologically-oriented children and youth studies in the Pacific mainly covering child-rearing practices, acculturation and child socialization - but it also has the merit of bringing such scholarship in conversation with the - predominantly European and North American - tradition of interpretive studies of children health and cultural production, and in particular with the child-centred perspective pioneered by the New Social Studies of Childhood at the end of the past century.

Writing from an anthropological standpoint, this exceptionally well-written book, enriched by an array of beautiful illustrations of life at Tūrama School realized by the author, makes an important contribution to the scarce body of Aotearoa New Zealand school ethnographies. A preliminary bibliographic survey suggests indeed that this is the first anthropological inquiry, set in the Pacific context, into children's production of a cultural world within an educational institution.

The core aim of this conceptually dense work is to "unravel the way children's practices contribute to the coproduction of their health through their engagement with and transformation of the social structures that guide and constrain children's practices" (9). It is interesting to note how such a question, which is not directed at a discrete phenomenon but rather at a whole universe of social-cultural practices, immediately positions the researcher's gaze at the interface between different analytical planes: the global and the local, the macro-structural and the microinteractional, the biological and the cultural. It is precisely the author's ability to dwell at this interface and translate the complexity of the ethnographic encounter into an intelligible narrative informed by the up-to-date, relevant literature that makes The Children in Child Health such a compelling and insightful read.

The first two chapters draw the research context by describing the socio-economic and ethnic composition of Papakura and retracing the colonial and postcolonial trajectories behind the 
constellation of social symptoms plaguing the predominantly Māori and Pasifika population that inhabit the low-income area of the suburb where Türama School is located, with a particular focus on rheumatic fever, its social causes and consequences, and the way it becomes structurally integrated into children's lives. In this context, the school represents a fundamentally caring environment and an outpost for the State's social, educational and healthcare interventions targeting the more impoverished segments of the urban population; but the school is also a space deputed to the production of a model of childhood, which can at times become fraught with contradictions. Already in this first part of the book, the reader is given a glimpse into the experiential world of the children of Tūrama, as the author interweaves her historical excursus with a portray of the children's coping strategies both with this structural violence and with their overexposure to public and media discourse on child health - strategies that are seamlessly embedded in the daily interactional routines of the school life and often acquire a ludic dimension.

Chapter 3 focuses on the issues of method and positioning in participated research with children. Here Spray examines different contemporary methodologies and the degree to which they construct the researcher's role along a scale that has the "acceptance" and the "rejection" of adultness at its extremes; she assesses their limits and proposes the "transparent guest" as "an operational mode for fieldwork" (48). As the author reflects on her own experience of negotiating her role in the classroom, she stresses how this eminently relational work implicates handling oneself amid the conundrums arising whenever there is a conflict between the different facets that the researcher's identity reveals on the field - observer, friend, depositary of the adult authority... These roles all demand different pedagogical stances and come with a burden of selfperceived and publicly assigned obligations towards oneself and others. The choices that one makes in these situations are never entirely strategic in a utilitarian sense but rather affectively charged and replete with ethical and pragmatic implications: it is at these turning points, where action can disclose or foreclose (sometimes permanently) networks of relationships with the research participants, that the fieldwork reveals its generative potentiality, which Spray effectively captures in her writing as she chooses to broaden the reader's horizon and embrace these episodes instead of expunging them from her ethnography.

Over the following five chapters, the author unpacks and dissects the ethnography's main subject, starting in Chapter 4 with an investigation of the relational and symbolic work unfolding at the school rheumatic fever prevention clinic, a space organized by pedagogic relations alternative to those enforced in the classroom that is "appropriated" by children "into strategies of relationshipbuilding" (68). Detailed renderings of interactional sequences transcribed from recordings and fieldnotes provide the point of departure for analysing the implicit aetiology of rheumatic fever and how it relates to the children's movements inside the discursive and practice field created by health interventions in the school context.

Each chapter remarkably engages in a dialogue with different bodies of literature to highlight different dimensions of the coproduction of child health: a discursive perspective on the interlacing between children's dietary patterns at school and the neoliberal rhetoric of parental responsibility (Chapter 5 ) is thus followed by a phenomenologically-oriented analysis of the children's incarnate experience of "sore throat", "hunger" and "cold" (Chapter 6). As this analytical trajectory draws closer to the experiential territory extending within the school walls, it is crossed by a palpable anti-reductionist tension in the always well-argued juxtaposition of anthropological/sociological theories of embodiment, interpretive approaches to peer culture, and socio-biological models of corporeal microprocesses. 
The last chapters elaborate more on the issue of children's encounters with illness and death. In Chapter 7, the author makes significant steps towards a relational theory of affective states by analysing how children's vulnerabilities are codified "through silence and touch, and resilience [is] practiced through bodily power, agency, and autonomy, through the embodiment of sore throat and its vigilant care, the language of aggression, or the shared language of pain" (151). Here, Spray's deep engagement with the field opens a window overlooking one of the most intimate corners of children's peer culture that is often excised from the adult conscience: the sphere of self-harm practices. The author's insightful analysis sheds light on how the rituals of concealment and display of the bodily traces of self-harm fit within a collective work of making affective wounds visible "beyond the capacity of language" (160).

In Chapter 8, the anthropologist delves into the still little-explored theme of children's perspectives on death, with a focus on how "death creeps unsummoned into everyday childhoods" (167). In what is perhaps the most hermeneutically-influenced part of the work, Spray observes how the "small talk of death", spatially and temporally removed from the death event, can take up many semiotic and affective shades and serve a variety of social and cultural objectives.

The multifaceted nature of Spray's research makes The Children in Child Health a valuable resource for a vast and heterogeneous audience: from social scientists researching across the fields of child studies, health policy and Pacific studies to teachers, educators and policy analysts working in a child-centred perspective; from graduate students looking for a vade mecum on finegrained childhood qualitative inquiry to veteran anthropologists and sociologists interested in deepening their knowledge of the context of Aotearoa New Zealand.

Among this book's many qualities, it is impossible not to mention its transparent rendering of the relationship between theory, method and data, which allows the reader to inspect the conceptual architecture of the study and critically engage with its different junctures, thereby opening the possibility for generative dialogue. Written in a terse prose that reconciles the virtue of epistemological rigour with the beauty of a well-crafted narrative, Spray's book is a graceful portrait of the collective life of a group of children and adults sharing a portion of their lives within the school walls which reconnects us with the core dimension of social research as an essentially collaborative endeavour. 